\title{
疏水链长对聚氧乙烯失水山梨醇脂肪酸酯界面扩张流变性质的影响
}

\author{
李 明 ${ }^{1,2}$ 曹 冲 ${ }^{1}$ 熊可洁 ${ }^{1}$ 杜凤沛 ${ }^{1, *}$ \\ ('中国农业大学理学院应用化学系, 北京 100193; 2黑龙江省农业科学院农药应用研究中心, 哈尔滨 150086)
}

摘要: 利用悬挂滴方法研究了系列聚氧乙烯失水山梨醇脂肪酸酯(TweenX)在正癸烷-水界面的扩张流变性 质. 实验结果表明, 疏水烷基链长较短的 Tween20 分子在界面上吸附量较大, 分子排列更紧密, Tween 40 和 Tween60 具有大致相同的 “有效截面积”, 导致饱和吸附时界面张力 $\left(\gamma_{\mathrm{cmc}}\right)$ 比较接近. TweenX浓度大于临界胶束 浓度 $(\mathrm{cmc})$ 时, 由于 Tween20 分子排列的更加紧密, 模量和弹性大于 Tween 40 和 Tween60. 当 TweenX的疏水 烷基链长达到一定长度时, TweenX的界面膜性质受疏水链长的影响减弱, Tween40 和 Tween60 的扩张参数相 差不大.

关键词：聚氧乙烯失水山梨醇脂肪酸酯； 疏水链长；界面； 扩张模量；相角 中图分类号: 0647

\section{Effect of Hydrophobic Chain Length on the Interfacial Dilational Rheological Properties of Polyoxyethylene Sorbitan Fatty Acid Ester}

\author{
LI Ming ${ }^{1,2} \quad$ CAO Chong ${ }^{1} \quad$ XIONG Ke-Jie ${ }^{1} \quad$ DU Feng-Pei ${ }^{1, *}$ \\ ('Department of Applied Chemistry, College of Science, China Agriculture University, Beijing 100193, P. R. China; \\ ${ }^{2}$ Research Center of Pesticide Application, Heilongjiang Academy of Agricultural Sciences, Harbin 150086, P. R. China)
}

\begin{abstract}
The dilational rheological properties of a series of polyoxyethylene sorbitan fatty acid surfactants (TweenX) at the decane/water interface have been investigated using the drop shape analysis method. The experiment results showed that larger quantities of the Tween20 molecules with shorter hydrophobic chain lengths were adsorbed at the interface because they could arrange more closely. Tween 40 and Tween60 molecules had similar "actual cross-sectional areas", which meant that their interfacial tensions were also similar at the critical micelle concentration $\left(\gamma_{\mathrm{cmc}}\right)$. Furthermore, when the bulk concentration exceeded the critical micelle concentration $(\mathrm{cmc})$, the modulus and elasticity of Tween20 were larger than those of Tween 40 and Tween60. When the hydrophobic alkyl chain of TweenX reached a certain length, the interfacial film properties of TweenX affected by the hydrophobic chain length decreased, which led to similar dilational parameters.
\end{abstract}

Key Words: Polyoxyethylene sorbitan fatty acid ester; Hydrophobic chain length; Interface;

Dilational modulus; Phase angle

\section{1 引言}

表面活性剂分子在流体界面上的吸附特性是 众多应用技术领域的理论基础. 近年来, 随着理论 模型和实验工作的不断发展, 表面活性剂界面扩张 流变性质的研究逐渐成为了流体界面性质研究的
重要手段. ${ }^{1-4}$ 通过扩张流变性质的研究, 可以获得界 面上分子的排列方式、分子间相互作用和界面聚集 体等信息, 这在理论和实践上均具有重要的意义. ${ }^{5-10}$

聚氧乙烯失水山梨醇脂肪酸酯(Tween $X, X$ 为 Tween 的名称代号) 是一类重要的多元醇非离子表

Received: September 16, 2014; Revised: December 1, 2014; Published on Web: December 1, 2014.

"Corresponding author. Email: dufp@cau.edu.cn; Tel: +86-10-62732507.

The project was supported by the Special Fund for Agroscientific Research in the Public Interest of China (201103024).

国家公益性行业(农业)科研专项(201103024)资助

(c) Editorial office of Acta Physico-Chimica Sinica 
面活性剂, 在多行业中广泛用作乳化剂、增溶剂和 润湿剂等. Tween $X$ 分子是以脂肪酸烷基长链作为 疏水基团, 以聚氧乙烯单元长链作为亲水基团, 根 据脂肪酸部分的不同, TweenX具有不同的型号. 目 前有关 TweenX扩张流变性质的研究主要集中在某 个型号的 TweenX 与其它表面活性物质混合吸附膜 特性, 如 Girardet 等 ${ }^{11}$ 研究了 Tween20 与 $\beta$-酪蛋白混 合膜在油水界面吸附特性; Ruiz-Henestrosa 等 ${ }^{12}$ 研 究了 Tween 20 与大豆球蛋白混合吸附膜和发泡特 性; RodriguezNino 等 ${ }^{13,14}$ 研究了牛血清白蛋白(BSA) 与 Tween 20 在乙醇水溶液和蔗糖水溶液表面的吸附 膜性质等.

本文利用悬挂滴方法研究了系列 TweenX在正 癸烷/水界面的扩张流变性质, 考察了疏水烷基链长 对 TweenX界面扩张流变性质的影响, 旨在为该系 列表面活性剂的实际应用提供理论基础.

\section{2 理论基础 ${ }^{15,16}$}

当界面面积发生周期性压缩和扩张时, 界面张 力也随之发生周期性变化, 扩张模量 $(\varepsilon)$ 定义为界面 张力 $(\gamma)$ 变化与相对界面面积 $(A)$ 变化的比值, 即:

$$
\varepsilon=\frac{\mathrm{d} \gamma}{\mathrm{d} \ln A}
$$

对于纯弹性界面, 界面张力的周期性变化与界 面面积的周期性变化是一致的; 但对于粘弹性界 面, 界面张力的周期性变化与界面面积的周期性变 化之间存在一定的相位差 $(\theta)$, 称为扩张模量的相 角. 扩张模量可写为复数形式:

$$
\varepsilon=\varepsilon_{\mathrm{d}}+\mathrm{i} \omega \eta_{\mathrm{d}}
$$

其中, $\varepsilon_{\mathrm{d}}$ 为扩张弹性, 也称为储存模量, 反映粘弹性 界面的弹性部分的贡献; $\omega \eta_{\mathrm{d}}$ 为扩张粘性模量, 也称 为损耗模量, 反映粘弹性界面的粘性部分的贡献; $\eta_{\mathrm{d}}$ 为扩张粘度; $\omega$ 为界面面积正弦变化的频率.

通过实验测得扩张模量的绝对值和相角可以 分别按式(3)和式(4)计算出扩张弹性 $\varepsilon_{\mathrm{d}}$ 和粘度 $\eta_{\mathrm{d}}$ :

$$
\begin{aligned}
& \varepsilon_{\mathrm{d}}=|\varepsilon| \cos \theta \\
& \eta_{\mathrm{d}}=\frac{|\varepsilon|}{\omega} \sin \theta
\end{aligned}
$$

\section{3 实验部分}

\section{1 实验样品及试剂}

系列 TweenX购自 Sigma Aldrich 公司(德国), 纯 度 $\geqslant 90 \%$. 分子结构见图 1. 相关物理化学性质 ${ }^{17}$ 列于

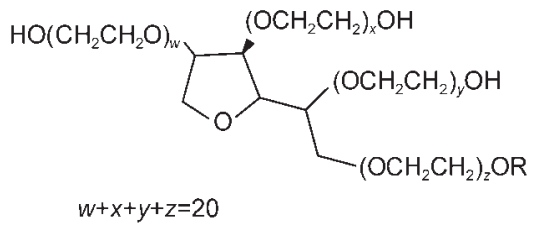

图 1 TweenX 的结构

Fig.1 Structures of TweenX

Tween20: $\mathrm{R}=-\mathrm{COC}_{11} \mathrm{H}_{23}$; Tween40: $\mathrm{R}=-\mathrm{COC}_{15} \mathrm{H}_{31}$;

Tween60: $\mathrm{R}=-\mathrm{COC}_{17} \mathrm{H}_{35}$

表 1. 从表 1 可以看出, 系列 TweenX 具有相同的氧 乙烯数, 随着疏水烷基链长的增加, TweenX 的亲油 亲水平衡(HLB)值逐渐减小, 说明烷基链越长, 整个 TweenX 分子更加亲油. 正癸烷(decane), 分析纯, 亦 购自 Sigma Aldrich 公司. 水为二次蒸馏去离子水.

\section{2 实验仪器及方法}

采用德国 Data Physics 公司生产的 OCA20 视频 光学接触角测量系统的扩展功能 ODG20 界面粘弹 测量仪测量. 实验开始时, 在正癸烷相中新注射一 个体积为 $6 \mu \mathrm{L}$ 的表面活性剂水溶液液滴, $10 \mathrm{~s}$ 后, 开始对液滴施加频率为 $0.1 \mathrm{~Hz}$ 的正弦面积形变扰 动, 摄像机实时拍摄记录液滴面积的瞬间变化, 通 过软件 SCA20 分析处理得到整个吸附过程的动态 界面张力和动态扩张参数, 直到界面张力达到完全 平衡为止, 之后在工作频率为 $0.005-0.2 \mathrm{~Hz}$ 进行变 频扩张粘弹性测量, 如图 2 所示. 整个实验温度控制 在 $(30.0 \pm 0.1){ }^{\circ} \mathrm{C}$, 扩张形变率 $(\Delta A / A)$ 为 $10 \%$. 正癸烷 为油相, 水相为不同浓度的 TweenX溶液.

\section{4 结果与讨论}

\subsection{TweenX的稳态界面张力}

图 3 为系列 TweenX的稳态界面张力. 从图 3 可 以看出, 随着疏水烷基链长的增加, TweenX界面张 力 $(\gamma)$ 开始明显降低时的浓度逐渐减小. Tween20 在 $1 \times 10^{-5} \mathrm{~mol} \cdot \mathrm{L}^{-1}$ 时 $\gamma$ 开始明显降低; Tween 40 在 $3 \times 10^{-6}$ $\mathrm{mol} \cdot \mathrm{L}^{-1}$ 时 $\gamma$ 开始明显降低; Tween60 在 $1 \times 10^{-6} \mathrm{~mol} \cdot$ $\mathrm{L}^{-1}$ 时 $\gamma$ 即开始明显降低. 这使 TweenX产生的临界胶 束浓度 $(\mathrm{cmc})$ 逐渐减小, 表明 TweenX 的疏水烷基链

\section{表 1 TweenX的物理化学性质}

Table 1 Physical chemistry properties of TweenX

\begin{tabular}{cccccc}
\hline \multirow{2}{*}{ Abbreviation } & Fatty acid & $n_{1}(\mathrm{C})^{\mathrm{a}}$ & $n_{2}(\mathrm{EO})^{\mathrm{b}}$ & $\begin{array}{c}\text { Average molecular } \\
\text { weight }\end{array}$ & HLB $^{\mathrm{c} 17}$ \\
\hline Tween20 & lauric acid & 12 & 20 & 1226 & 16.7 \\
Tween40 & palmitic acid & 16 & 20 & 1287 & 15.6 \\
Tween60 & stearic acid & 18 & 20 & 1312 & 14.9 \\
\hline
\end{tabular}

${ }^{\mathrm{a}}$ carbon numbers of fatty acid chain; ${ }^{\mathrm{b}}$ average numbers of ethylene oxide (EO) block; ' HLB: hydrophile lipophile balance 


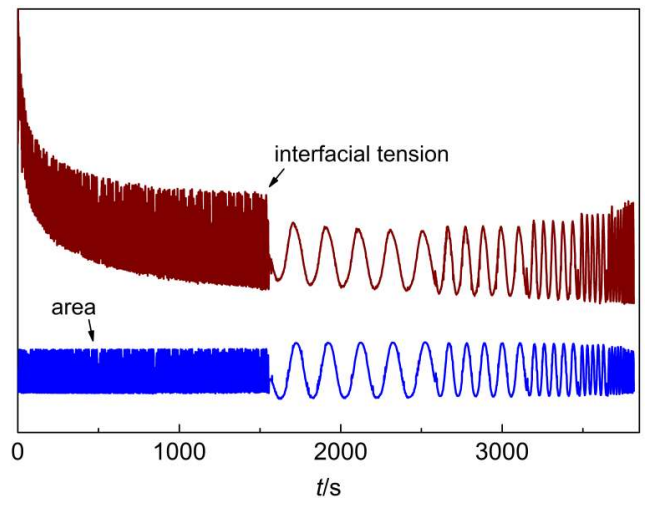

图 2 TweenX界面扩张流变性质测量

Fig.2 Measurement of interfacial dilational rheological properties of TweenX

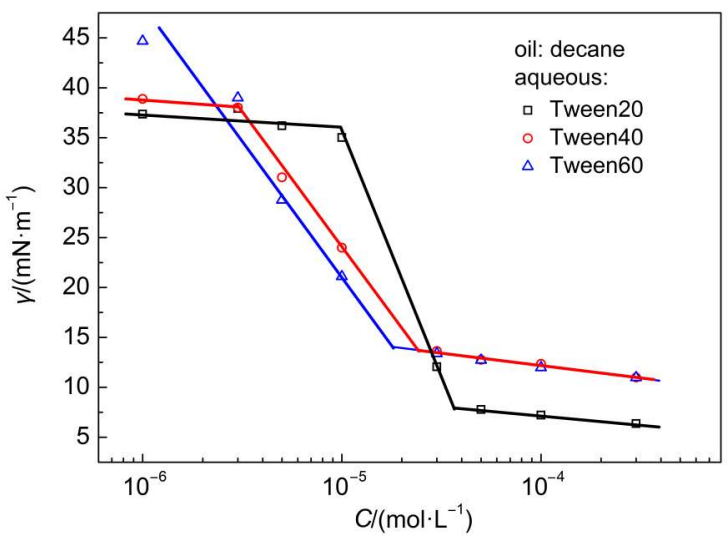

图 3 TweenX的稳态界面张力 $(\gamma)$

Fig.3 Equilibrium interfacial tension $(\gamma)$ for TweenX

长越长, 在体相中形成胶束的能力越强.

需要指出的是, 随着体相浓度的增加, Tween20 和 Tween 40 稳态界面张力曲线上出现两个明显的拐 点,一般来说, 这意味着表面活性剂在界面上吸附 饱和之前形成了胶束前聚集体. ${ }^{18}$ 随着疏水烷基链 长的增加, TweenX 界面张力曲线上低浓度拐点所 对应的浓度逐渐减小, 形成胶束前聚集体所对应的 浓度范围减小.

从图 3 可以获得一些界面活性参数, 分子截面 积 $\left(A_{\min }\right)$ 可以通过下面 Gibbs 方程(5)和公式(6)计算 得到

$$
\begin{aligned}
& \Gamma_{\text {max }}=\frac{-1}{(2.303 n R T)} \frac{\mathrm{d} \gamma}{\mathrm{d}(\lg C)} \\
& A_{\text {min }}=\frac{10^{18}}{N \Gamma_{\text {max }}}
\end{aligned}
$$

式中 $R$ 为理想气体常数, $T$ 为绝对温度, $n$ 取 $1, N$ 为 阿伏伽德罗常数, $\mathrm{d} \gamma / \mathrm{d}(\lg C)$ 为稳态界面张力曲线两 个拐点之间或一个拐点之前界面张力值线性拟合 的斜率. $A_{\text {min }}$ 计算结果、临界胶束浓度 $(\mathrm{cmc})$ 和饱和吸
附时界面张力 $\left(\gamma_{\mathrm{cmc}}\right)$ 列于表 2 .

从表 2 可以更明确的看出, 随着疏水烷基链长 的增加, TweenX 的 $\mathrm{cmc}$ 逐渐减小. 此外, 吸附饱和 时, Tween 20 的 $\gamma_{\mathrm{cmc}}$ 明显小于 Tween 40 和 Tween60, 这 与 Tween 20 的 $A_{\text {min }}$ 小于 Tween 40 和 Tween 60 的结果 是一致的. 说明 Tween 20 分子在界面上吸附量较大, 分子排列更紧密.

值得注意的是, Tween 60 的 $A_{\text {min }}$ 大于 Tween 40 的, 说明 Tween60 的吸附量较少, 但 $\gamma_{\mathrm{cmc}}$ 却与 Tween40 比较接近, 分别为 13.93 和 $13.73 \mathrm{mN} \cdot \mathrm{m}^{-1}$. Cao 等 ${ }^{19}$ 认为界面张力的降低取决于吸附在界面上 表面活性剂分子的“有效截面积”, “有效截面积”越 大, 界面张力越低. TweenX与一般的表面活性剂相 比具有较大的分子量和比较复杂的支链结构, 当分 子量足够大时, TweenX 分子自身的缠绕结构可能 使吸附在界面上表面活性剂分子的“有效截面积” 受疏水烷基链长的影响减弱, Tween 60 和 Tween 40 的“有效截面积”大致相同, 因此, 虽然 Tween60 界面 吸附量较少, 却与 Tween 40 具有比较接近的 $\gamma_{\mathrm{cmc}}$.

\subsection{Tween X 的动态界面张力及动态扩张性质}

图 4 为浓度 $1 \times 10^{-5} \mathrm{~mol} \cdot \mathrm{L}^{-1}$ 的系列 TweenX 的动 态界面张力和动态扩张性质. 从图 4(a) 可以看出, 随 着疏水烷基链长的增加, TweenX 分子的疏水作用 增强, 向界面扩散吸附的趋势增大. 实验时间内, Tween20 界面张力降低的拐点不明显, 在 $10000 \mathrm{~s}$ 之 后达到吸附平衡; Tween 40 在约 $1200 \mathrm{~s}$ 时界面张力 开始明显降低, 大约 $6000 \mathrm{~s}$ 时达到平衡; Tween60在 约 $120 \mathrm{~s}$ 时界面张力即开始明显降低, 大约 $3000 \mathrm{~s}$ 时 即达到平衡.

从图 4(b, c) 可以看出, Tween 40 和 Tween 60 的动 态界面扩张模量和扩张弹性随时间变化的转折点 与图 4(a) 界面张力曲线上的转折点并不一致, 扩张 模量和扩张弹性随着界面的老化, 阶梯增大至达到 平衡. 从图 4(d)还可以看出, 扩张粘性随时间的变化 不大, 且粘性均小于 $5 \mathrm{mN} \cdot \mathrm{m}^{-1}$, 说明此时界面以弹

表 2 TweenX 的 $\mathrm{cmc}, \gamma_{\mathrm{cmc}}$ 和 $A_{\text {min }}$

Table 2 cmc, $\gamma_{\mathrm{cmc}}$, and $A_{\min }$ of TweenX

\begin{tabular}{cccc}
\hline Abbreviation & $\mathrm{cmc} /\left(\mathrm{mol} \cdot \mathrm{L}^{-1}\right)$ & $\gamma_{\mathrm{cmc}} /\left(\mathrm{mN} \cdot \mathrm{m}^{-1}\right)$ & $A_{\min } / \mathrm{nm}^{2}$ \\
\hline Tween20 & $3.7 \times 10^{-5}$ & 7.94 & 1.11 \\
Tween40 & $2.4 \times 10^{-5}$ & 13.73 & 1.82 \\
Tween60 & $1.8 \times 10^{-5}$ & 13.93 & 2.54 \\
\hline
\end{tabular}

cmc: the critical micelle concentration; $\gamma_{\text {cmc }}$ interfacial tension at the critical micelle concentration; $A_{\min }$ : the minimum area per molecule at the decane-water interface 

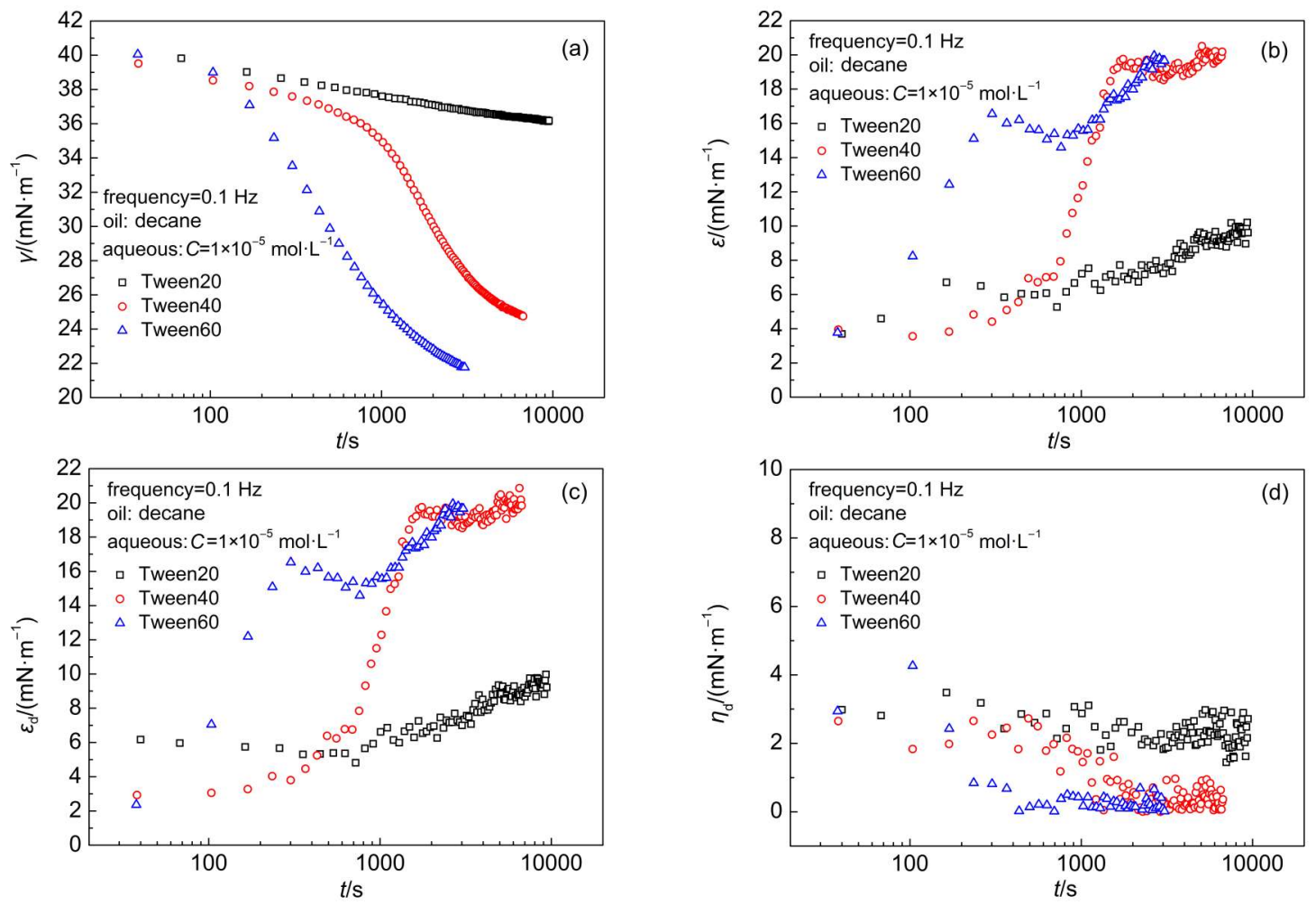

图 4 TweenX的动态界面张力和动态扩张流度性质

Fig.4 Dynamic interfacial tension and dilational rheological properties for TweenX

(a) interfacial tension, (b) modulus, (c) elasticity, (d) viscosity

性部分为主, 粘性较小. 一般认为, 扩张性质比界面 张力更能反映界面膜的状态 ${ }^{20}$ 出现这种现象的原 因可能是, 当体相浓度达到一定时, 先吸附的 Tween 40 和 Tween60 分子形成了粘性较小的“弹性 界面膜”, 阻碍了后续 Tween 40 和 Tween60 分子的吸 附,造成界面上出现剧烈的重排和取向等过程.

\section{3 工作频率对 TweenX 界面扩张性质影响}

扩张流变实验的工作频率是影响扩张参数的 重要因素. 扩张频率对不同浓度 TweenX扩张模量 及相角影响的变化趋势相似, 为简便起见, 仅以

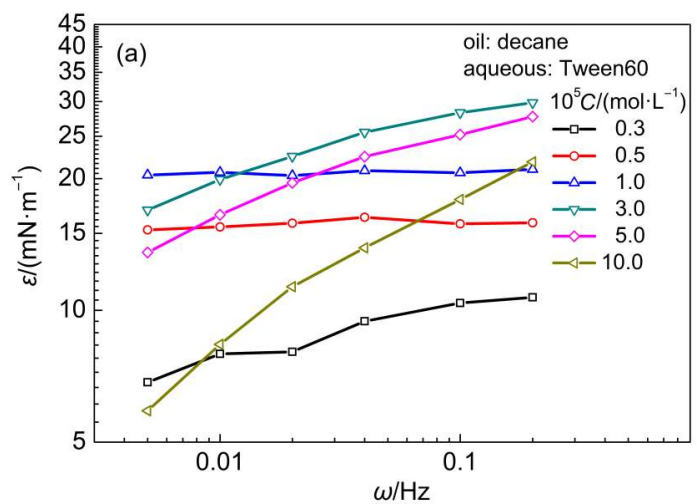

Tween60 为例进行讨论. 图 5 为扩张频率对不同浓 度 Tween60 扩张模量及相角影响. 扩张频率对不同 浓度 Tween 20 和 Tween 40 的扩张模量及相角影响见 Supporting Information. 从图 5 可以看出, 随着频率 增大, 扩张模量逐渐增大, 相角逐渐减小. 这是因 为, 频率较低时, Tween60 分子有足够的时间通过扩 散交换等弛豫过程来消除由界面形变所产生的界 面张力梯度, 扩张模量较小, 随着实验频率的逐渐 增大, 界面面积变化加快, 界面张力梯度来不及消 除, 扩张模量就较大. 因此, 扩张模量随着频率的增

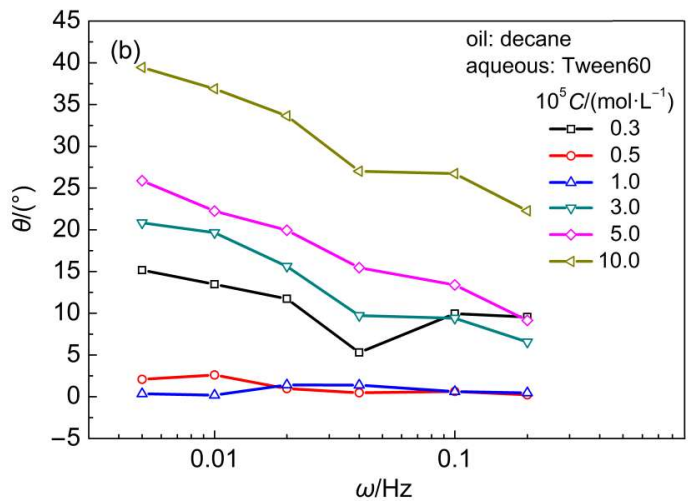

图 5 Tween60 的界面扩张模量(a)和相角(b)随工作频率的变化

Fig.5 Interface dilational modulus (a) and phase angle (b) as a function of frequency for Tween60 
大而增大. 另一方面, 随着频率的增大, 弛豫过程对 模量的贡献减小导致了相角减小.

扩张粘弹参数随频率的变化规律反映了界面 膜的特性. 当界面扩张实验的频率 $(0.2 \mathrm{~Hz})$ 远远小于 弛豫过程的特征频率时, 扩张模量对频率的双对数 曲线几乎是线性关系. 不同弛豫过程曲线的斜率范 围不同, 如扩散弛豫过程(符合 Lucassen-Tempel 模 型)的斜率小于 0.5 , 界面上存在特殊结构时, 弛豫过 程极限斜率为 1 . 斜率越小, 则界面膜的弹性部分所 占的比例越大. ${ }^{21}$

图 6 为不同浓度 TweenX 的 $\lg |\varepsilon|-\lg \omega$ 曲线的斜 率, 从图 6 可以看出, 在低浓度时, $\lg |\varepsilon|-\lg \omega$ 曲线斜 率随着 TweenX 浓度的增加逐渐减小, Tween 40 和 Tween60 形成了最低值平台; 当浓度大于 $\mathrm{cmc}$ 时, $\lg |\varepsilon|-\lg \omega$ 曲线斜率明显增大. 需要指出的是, 这种 非离子表面活性剂 $\lg |\varepsilon|-\lg \omega$ 曲线斜率随浓度增大 先减小后增大的现象与一般的离子型表面活性剂 有着明显的不同. 一般而言, 离子型表面活性剂 $\lg |\varepsilon|-\lg \omega$ 曲线斜率均随着浓度的增加而单调增 大. ${ }^{22}$ 说明界面膜的弹性部分所占的比例在逐渐减 小, 体相与界面间的扩散交换作用在逐渐增强. 而 在本研究中, 非离子型表面活性剂界面膜弹性部分 所占的比例在低浓度时却在逐渐增强, 当体相浓度 大于 $\mathrm{cmc}$ 时, 体相与界面间的扩散交换作用才逐渐 增强. 出现这种现象的原因是非离子表面活性剂亲 水部分的氧乙烯链明显大于离子型表面活性剂的 亲水部分, 导致其在界面与体相之间扩散交换的特 征时间较长, 同时, 吸附在界面上的非离子表面活 性剂分子之间的相互作用也更强. 因此, 在 $\mathrm{cmc}$ 之 前, TweenX浓度的增加主要增加了界面上的表面

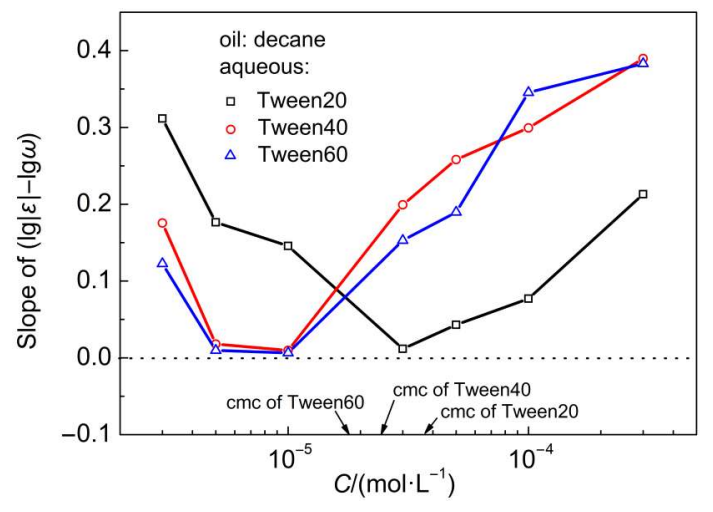

图 6 TweenX浓度对 $\lg |\varepsilon|-\lg \omega$ 曲线斜率的影响

Fig.6 Influence of Tween $X$ concentrations on slope of $\lg |\varepsilon|-\lg \omega$ curves
活性剂分子浓度, 使界面吸附膜分子排列更紧密, 膜的弹性增强, $\lg |\varepsilon|-\lg \omega$ 曲线斜率出现最低值或最 低值平台. 当体相浓度大于 $\mathrm{cmc}$ 时, 界面附近的胶束 与界面之间的交换明显加快, 粘性部分对模量的贡 献增大, $\lg |\varepsilon|-\lg \omega$ 曲线斜率才明显增大.

从图 6 还可以看出, TweenX 经过最低值平台或 最低值时, $\lg |\varepsilon|-\lg \omega$ 曲线的斜率接近 0 , 此时, 扩张 模量几乎不随着扩张频率的变化而变化, 说明此时 界面由较慢的弛豫过程控制, 其特征频率远远小于 实验频率. 从图 5(b)中也可以看出, 相角的实验结果 与斜率的实验结果是一致的, 对应双对数曲线斜率 最小值平台或最小值时的浓度, 相角接近 $0^{\circ}$, 此时 界面膜粘性部分所占比例非常小, 界面接近纯弹性 膜. Tween 40 和 Tween 60 经过 $\lg |\varepsilon|-\lg \omega$ 曲线斜率最 低值平台时的浓度明显小于 Tween 20 达到最低值时 的浓度, 这是由于 Tween 40 和 Tween 60 的疏水烷基 链长较长, 分子更加疏水, 在界面上吸附的更加牢 固, 此外 Tween 40 和 Tween 60 的 $\mathrm{cmc}$ 也较小, 在较低 浓度便可形成胶束增加界面粘性, 因此 Tween 40 和 Tween60 在较低浓度便可形成接近纯弹性界面膜.

\section{4 浓度对 TweenX界面扩张性质影响}

界面扩张模量的产生是由于界面和界面附近 存在的微观弛豫过程. 因此, 界面扩张参数可以反 映出界面分子之间的相互作用和界面吸附层结构 的信息. ${ }^{23}$ 低分子量表面活性剂主要存在分子在界 面与体相之间的扩散交换和分子在界面层中的构 象变化两种弛豫过程. ${ }^{24}$ 而对于较大分子量表面活 性剂而言, 扩散交换的特征时间较长, 因此界面与 体相之间的扩散交换可忽略不计, 但是必须要考虑 到界面上不同区域之间的分子交换. ${ }^{25}$ 表面活性剂 体相浓度的增加对界面扩张模量有两方面的影响: 一方面增大了界面上表面活性剂分子的浓度, 导致 界面分子间更强的相互作用, 界面膜的扩张模量增 大; 另一方面也增大了从体相向新生成界面通过扩 散补充表面活性剂分子的能力, 这有利于重新恢复 平衡, 会降低界面模量. 上述这两种因素的共同作 用导致扩张模量随浓度的增加通过一个极大值. ${ }^{26}$

我们在液液界面的扩张流变实验中发现, 即使 干净的癸烷/水界面也会有较小的模量和相角. 从动 态扩张性质, 即图 4 中也可以看出, 在短时间时, TweenX在界面上的吸附量较低, 不能形成界面膜, 但模量和相角却并不为零. 江龙等 ${ }^{27}$ 认为这是界面 上存在着一种由色散力相吸引起的对界面压敏感 

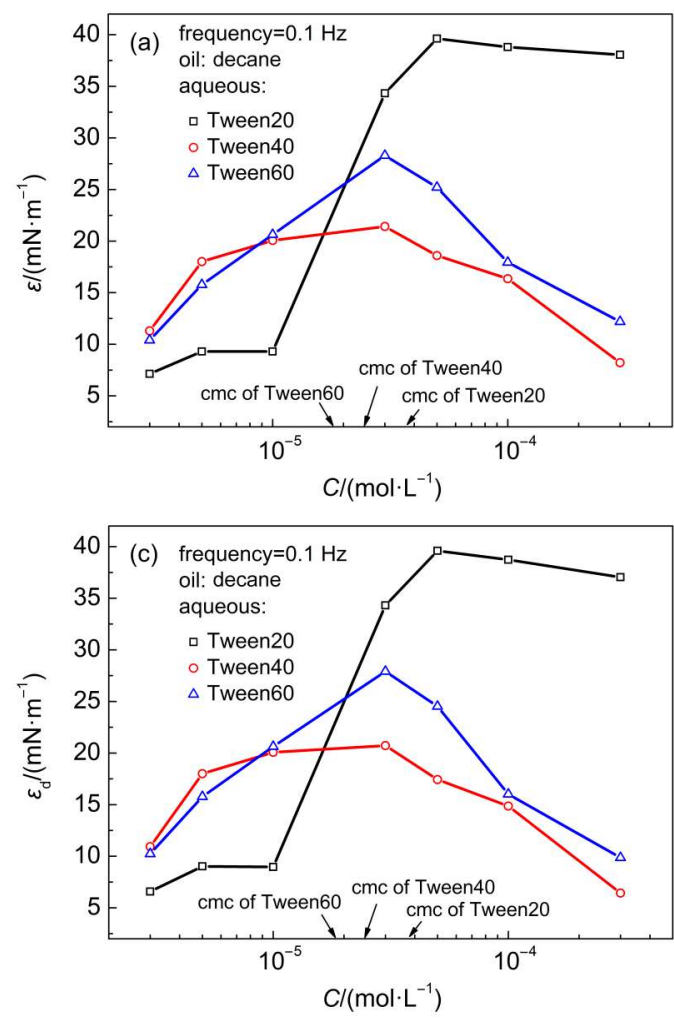
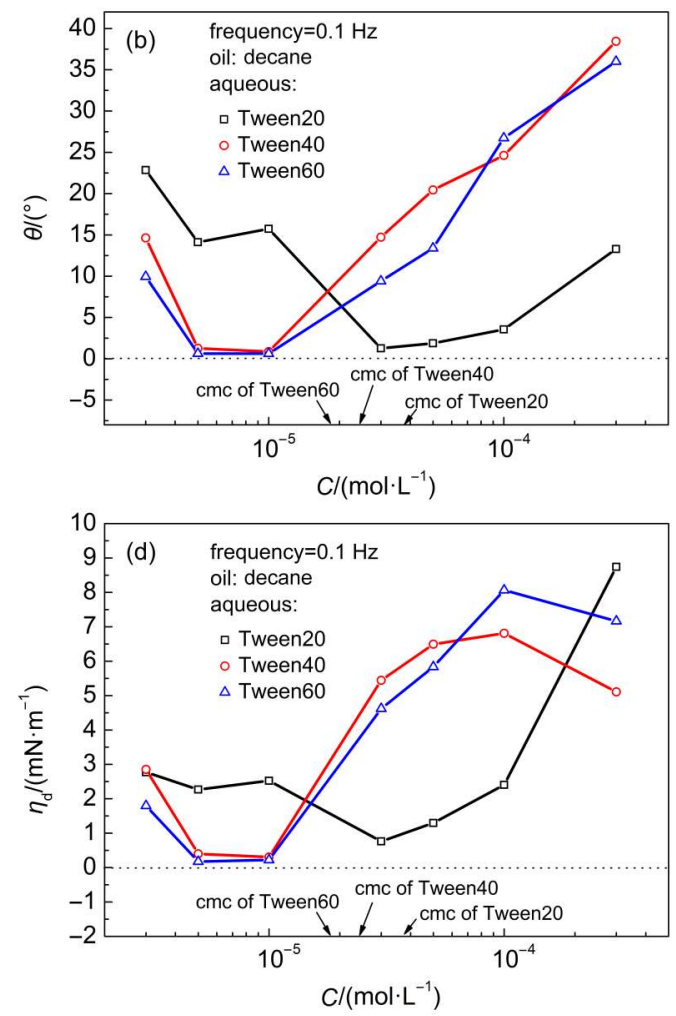

图 7 TweenX界面扩张流变性质随浓度的变化

Fig.7 Interfacial dilational rheological properties as a function of concentration for TweenX

(a) modulus, (b) phase angle, (c) elasticity, (d) viscosity

的“界面缔合物”, 在液-液界面上, 即使没有表面活 性剂存在, 也会有界面张力的下降或界面压的升高.

工作频率为 $0.1 \mathrm{~Hz}$ 时, TweenX 的界面扩张模 量、相角、弹性和粘性随浓度的变化趋势如图 7 所 示. 从图 7 可以看出, 当 TweenX浓度为 $1 \times 10^{-6} \mathrm{~mol}$. $\mathrm{L}^{-1}$ 时, 吸附在界面上的表面活性剂非常少, TweenX 与“界面缔合物”混合吸附在界面上. 随着 TweenX 浓度的增加, 表面活性剂界面膜逐渐形成, “界面缔 合物”逐渐消失, 由于 TweenX 的分子量较大, 其在 界面与体相之间的分子扩散交换特征时间较长, 体 相浓度增加主要增加了界面上表面活性剂分子浓 度, 这增强了界面上表面活性剂分子之间的相互作 用. 因此, 在低于 $\mathrm{cmc}$ 时, 随着浓度的增加, 模量和弹 性逐渐增大, 而相角和粘性变化的趋势为逐渐降低 至最低值或达到最低值平台. 当 Tween $\mathrm{X}$ 浓度大于 $\mathrm{cmc}$ 时, 随着浓度的增加, 界面附近的胶束与界面之 间的扩散交换逐渐增强, 相角逐渐增大, 如前面所 讨论的, 扩张模量经过极大值.

需要指出的是, TweenX 在 cmc 之前, 相角达到 的最低值或最低值平台接近 $0^{\circ}$, 此时的粘性也几乎 为零, 说明界面膜以弹性为主, 与上文所述 $\lg |\varepsilon|-$ $\lg \omega$ 曲线斜率的结果是一致的. 此外, 前文所述图 4
中, 当 Tween40 和 Tween60 浓度为 $1 \times 10^{-5} \mathrm{~mol} \cdot \mathrm{L}^{-1}$ 时, 扩张弹模量和弹性曲线与界面张力曲线转折点 的不一致性也说明, 当形成弹性较强界面膜时, Tween40 和 Tween60 浓度的继续增大, 导致了分子 在界面上出现较复杂的重排和取向等过程.

从图 7 还可以看出, TweenX 浓度小于 $\mathrm{cmc}$ 时, Tween40 和 Tween60 分子具有较长疏水烷基链, 可 能是烷基碳链的缠绕折叠使分子间存在较强的相 互作用, 导致 Tween40 和 Tween60 扩张模量和扩张 弹性大于 Tween20, 相角和粘性小于 Tween 20; TweenX 浓度大于 $\mathrm{cmc}$ 时, 疏水烷基链长较短的 Tween20 分子在界面上饱和吸附时, 排列的更加紧 密, 导致模量和弹性大于 Tween 40 和 Tween 60 , 相角 和粘性小于 Tween 40 和 Tween60. 有趣的是, Tween 40 和 Tween 60 的扩张参数相差不大, 这可能是当 TweenX 的疏水烷基链长达到一定长度时, TweenX 的界面膜性质受疏水链长的影响减弱, 界面膜粘弹 性质可能是由亲水部分控制.

\section{5 结 论}

研究了疏水烷基链长不同的系列聚氧乙烯失 水山梨醇脂肪酸酯 Tween 20、Tween40 和 Tween60 在 
癸烷-水界面的扩张流变性质. 研究发现, TweenX界 面饱和吸附时, 疏水烷基链长较短的 Tween20 分子 在界面上吸附量较大, 分子排列更紧密. 当 TweenX 分子量足够大时, 分子自身的缠绕结构可能使吸附 在界面上表面活性剂分子的“有效截面积”受疏水 烷基链长的影响减弱, 使 Tween60 和 Tween40 的“有 效截面积” 大致相同, Tween 40 与 Tween 60 的 $\gamma_{\mathrm{cmc}}$ 比较 接近. TweenX浓度小于 $\mathrm{cmc}$ 时, 形成了近纯弹性的 界面膜, 疏水烷基链较长的 Tween 40 和 Tween60 分 子的缠绕折叠使分子间存在较强的相互作用, 导致 Tween 40 和 Tween 60 扩张模量和扩张弹性大于 Tween20. 当 TweenX浓度大于 $\mathrm{cmc}$, Tween20 分子在 界面上饱和吸附时, 排列的更加紧密, 模量和弹性 大于 Tween 40 和 Tween 60 . 当 TweenX 的疏水烷基链 长达到一定长度时, TweenX的界面膜性质受疏水 链长的影响减弱, 导致 Tween 40 和 Tween60 的扩张 参数相差不大.

Supporting Information: available free of charge via the internet at http://www.whxb.pku.edu.cn.

\section{References}

(1) Zholkovskij, E. K.; Kovalchuk, V. I.; Fainerman, V. B.; Loglio, G.; Kragel, J.; Miller, R.; Zholob, S. A.; Dukhin, S. S. J. Colloid Interface Sci. 2000, 224, 47. doi: 10.1006/jcis.1999.6669

(2) Noskov, B. A. Curr. Opin. Colloid Interface Sci. 2010, 15, 229. doi: 10.1016/j.cocis.2010.01.006

(3) Kovalchuk, V. I.; Makievski, A. V.; Kragel, J.; Pandolfini, P.; Loglio, G.; Liggieri, L.; Ravera, F.; Miller, R. Colloids Surf. A-Physicochem. Eng. Asp. 2005, 261, 115. doi: 10.1016/j. colsurfa.2004.12.051

(4) Benjamins, J.; Lyklema, J.; Lucassen-Reynders, E. H. Langmuir 2006, 22, 6181. doi: 10.1021/la060441h

(5) Wang, Y. Y.; Zhang, L.; Sun, T. L.; Fang, H. B.; Zhao, S.; Yu, J. Y. Acta Phys. -Chim. Sin. 2003, 19, 297. [王宜阳, 张 路, 孙 涛垒, 方洪波, 赵 濉, 俞稼镛. 物理化学学报, 2003, 19, 297.] doi: 10.3866/PKU.WHXB20030403

(6) Romoscanu, A. I.; Mezzenga, R. Langmuir 2005, 21, 9689. doi: $10.1021 / \mathrm{la} 051241 \mathrm{t}$

(7) El-Sadek, R. I.; Roushdy, M.; Magda, J. J. Langmuir 2007, 23, 7907. doi: 10.1021/la700864k

(8) Huang, Y. P.; Zhang, L.; Luo, L.; Zhang, L.; Zhao, S.; Yu, J. Y. Acta Phys. -Chim. Sin. 2007, 23, 12. [黄玉萍, 张 否, 罗 澜, 张 路, 赵 濉, 俞稼镛. 物理化学学报, 2007, 23, 12.] doi: 10.3866/PKU.WHXB20070103

(9) Zhang, L.; Wang, X. C.; Gong, Q. T.; Luo, L.; Zhang, L.; Zhao, S.; Yu, J. Y. Acta Phys. -Chim. Sin. 2007, 23, 1881. [张＼cjkstart否,
王晓春, 宫清涛, 罗 澜, 张 路, 赵 濉, 俞稼镛. 物理化学学 报, 2007, 23, 1881.] doi: 10.3866/PKU.WHXB20071210

(10) Zhu, Y. Y.; Xu, G. Y. Acta Phys. -Chim. Sin. 2009, 25, 191. [朱艳艳, 徐桂英. 物理化学学报, 2009, 25, 191.] doi: 10.3866/ PKU.WHXB20090135

(11) Girardet, J. M.; Humbert, G.; Creusot, N.; Chardot, V.; Campagna, S.; Courthaudon, J. L.; Gaillard, J. L. J. Colloid Interface Sci. 2001, 243, 515. doi: 10.1006/jcis.2001.7893

(12) Ruiz-Henestrosa, V. P.; Sanchez, C. C.; RodriguezPatino, J. M. Ind. Eng. Chem. Res. 2008, 47, 2876. doi: 10.1021/ie071518f

(13) RodriguezNino, M. R.; Wilde, P. J.; Clark, D. C.; Husband, F. A.; RodriguezPatino, J. M. J. Agric. Food Chem. 1997, 45, 3010. doi: $10.1021 /$ jf9701143

(14) RodriguezNino, M. R.; Wilde, P. J.; Clark, D. C.; Husband, F. A.; RodriguezPatino, J. M. J. Agric. Food Chem. 1997, 45, 3016. doi: 10.1021/jf970115v

(15) Lucassen, J. J. Colloid Interface Sci. 1982, 85, 52. doi: 10.1016/ 0021-9797(82)90234-X

(16) Lucassen, J. Colloids and Surfaces 1992, 65, 139. doi: 10.1016/ 0166-6622(92)80269-8

(17) Liu, C.; Li, J. H.; Liu, B.; Sun, L. Y. The Surfactant Application Manual, 3nd ed.; Chemical Industry Press: Beijing, 2004; pp 39-46. [刘 程, 李江华, 刘 博, 孙丽燕. 表面活性剂应用 手册, 第三版. 北京: 化学工业出版社, 2004: 39-46.]

(18) Sakai, T.; Kaneko, Y.; Tsujii, K. Langmuir 2006, 22, 2039. doi: $10.1021 / \mathrm{la} 052640 \mathrm{r}$

(19) Cao, Y.; Zhao, R. H.; Zhang, L.; Xu, Z. C.; Jin, Z. Q.; Luo, L.; Zhang, L.; Zhao, S. Energy Fuels 2012, 26, 2175. doi: 10.1021/ ef201982s

(20) Huang, Y. P.; Zhang, L.; Zhang, L.; Luo, L.; Zhao, S.; Yu, J. Y. J. Phys. Chem. B 2007, 111, 5640. doi: 10.1021/jp070997t

(21) Prins, A.; Bos, M. A.; Boerboom, F. J. G.; van Kalsbeek, H. K. A. I. Proteins at Liquid Interfaces; Möbius, D., Miller, R., Eds.; Elsevier Sciene B. V.: Amsterdam, 1998; pp 341-384.

(22) Luo, L.; Wang, D. X.; Zhang, L.; Zhao, S.; Yu, J. Y. J. Dis. Sci. Technol. 2007, 28, 263. doi: 10.1080/01932690601059594

(23) Liggieri, L.; Miller, R. Curr. Opin. Colloid in Interface Sci. 2010, 15, 256. doi: 10.1016/j.cocis.2010.02.003

(24) Kopperud, H. B. M.; Hansen, F. K. Macromolecules 2001, 34, 5635.

(25) Noskov, B. A.; Akentiev, A. V.; Bilibin, A. Y.; Zorin, I. M.; Miller, R. Adv. Colloid Interface 2003, 104, 245. doi: 10.1016/ S0001-8686(03)00045-9

(26) Zhan, Q.; Zhou, Z. H.; Dong, L. F.; Wang, H. Z.; Cai, H. Y.; Zhang, F.; Zhang, L.; Zhang, L.; Zhao, S. Colloids Surf. A-Physicochem. Eng. Asp. 2014, 455, 97. doi: 10.1016/j. colsurfa.2014.04.031

(27) Zhu, H.; Tang, J. A.; Li, J. R.; Li, X. C.; Jiang, L.; Zhao, F. Chinese Science Bulletin 2002, 584. [ 朱 红, 唐季安, 李津 如, 李兴长, 江 龙, 赵 丰. 科学通报, 2002, 584.] 
Supplementary Information for Acta Phys. -Chim. Sin. 2015, 31 (2), 322-328

doi: 10.3866/PKU.WHXB201412012

\title{
疏水链长对聚氧乙烯失水山梨醇脂肪酸酯界面扩张流变性 质的影响
}

\author{
李 明 ${ }^{1,2}$ 曹 冲 ${ }^{1}$ 熊可洁 ${ }^{1}$ 杜风沛 ${ }^{1, *}$ \\ ( ${ }^{1}$ 中国农业大学理学院应用化学系, 北京 100193; 2 黑龙江省农业科学院农药应用研究中心, 哈尔滨 \\ 150086)
}

\section{Effect of Hydrophobic Chain Length on the Interfacial Dilational Rheological Properties of Polyoxyethylene Sorbitan Fatty Acid Ester}

\author{
LI Ming $^{1,2} \quad$ CAO Chong $^{1} \quad$ XIONG Ke-Jie $^{1} \quad$ DU Feng-Pei ${ }^{1, *}$ \\ ( ${ }^{1}$ Department of Applied Chemistry, College of Science, China Agriculture University, Beijing 100193, P. R. \\ China; ${ }^{2}$ Research Center of Pesticide Application, Heilongjiang Academy of Agricultural Sciences, Harbin \\ 150086, P. R. China)
}

*Corresponding author. Email: dufp@cau.edu.cn; Tel: +86-10-62732507. 

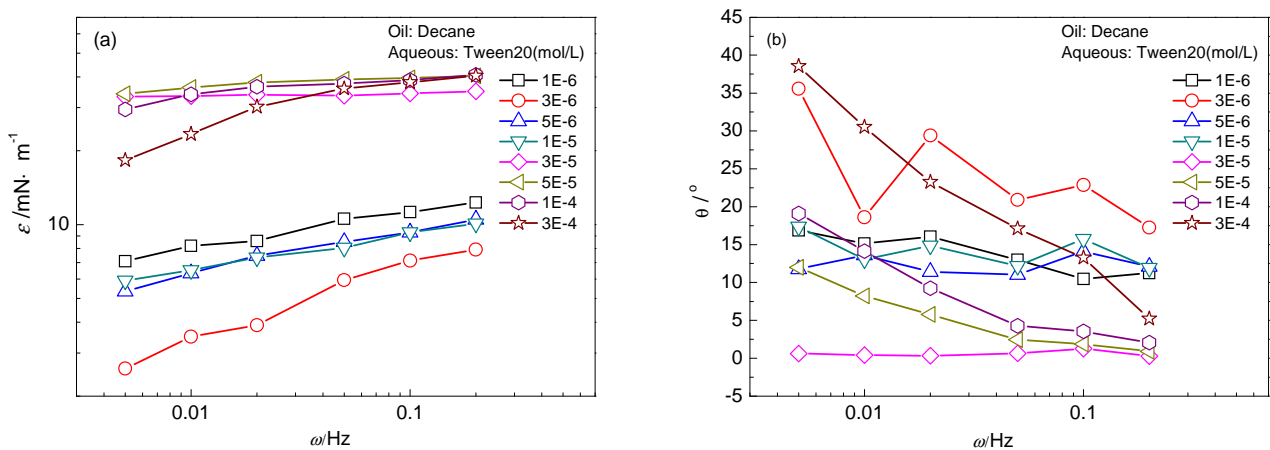

图 S1 Tween20 的界面扩张模量(a)和相角(b)随工作频率的变化

Fig.S1 Interface dilational modulus (a) and phase angle (b) as a function of frequency for Tween20
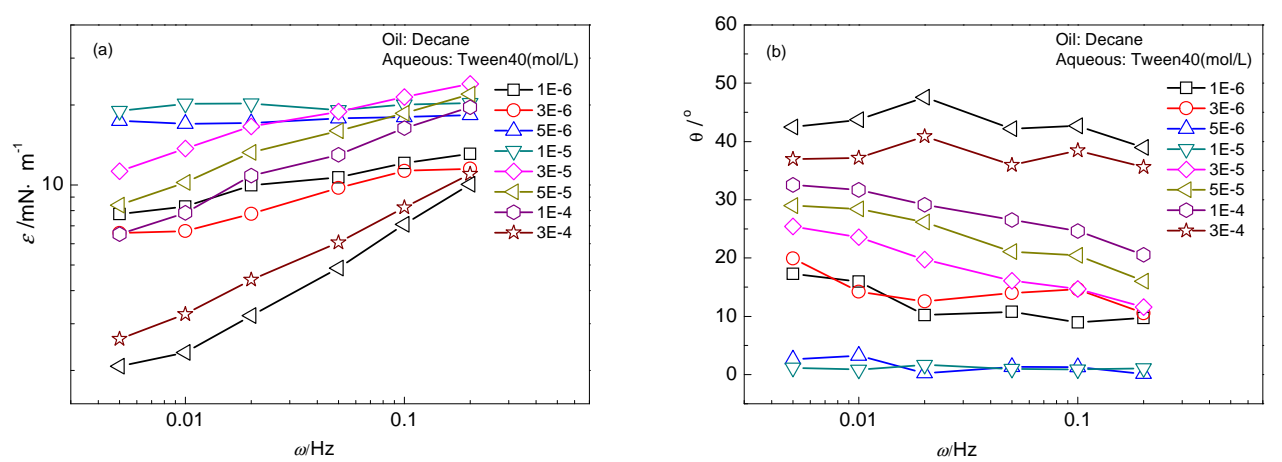

图 S2 Tween40 的界面扩张模量(a)和相角(b)随工作频率的变化

Fig.S2 Interface dilational modulus (a) and phase angle (b) as a function of frequency for Tween 40 